\title{
Reproducibility of intravoxel incoherent motion of liver on a 3.0T scanner: free-breathing and respiratory-triggered sequences acquired with different numbers of excitations
}

\author{
Andrzej Cieszanowski ${ }^{1 A, B, D, E, F}$, Katarzyna Pasicz ${ }^{2 A, B, C, D, E, F}$, Joanna Podgórska ${ }^{1,3 A, B, C, D, E, F}$, Ewa Fabiszewska 2A,B,D, \\ Witold Skrzyński2 ${ }^{2 A, C, D, E, F}$, Jakub Jasieniak ${ }^{1 A, B, C, D, F}$, Agnieszka Anysz-Grodzicka ${ }^{1 A, D}$, Iwona Grabska ${ }^{2 B, D}$, \\ Jakub Pałucki ${ }^{1 A, D}$, Martyna Naduk-Ostrowska ${ }^{1 A, B, D}$, Beata Jagielska ${ }^{A A, D}$, Paweł Kukołowicz ${ }^{2 A, B, D, E}$ \\ 'Department of Radiology I, Maria Skłodowska-Curie Memorial Cancer Center and Institute of Oncology, Warsaw, Poland \\ ${ }^{2}$ Medical Physics Department, Maria Skłodowska-Curie Memorial Cancer Center and Institute of Oncology, Warsaw, Poland \\ ${ }^{3} 2^{\text {nd }}$ Department of Clinical Radiology, Medical University of Warsaw, Warsaw, Poland \\ ${ }^{4}$ Department of Oncological Diagnosis and Cardio-Oncology, Curie Memorial Cancer Center and Institute of Oncology, Warsaw, Poland
}

\section{Abstract}

Purpose: To optimise the intravoxel incoherent motion (IVIM) imaging of the liver on a 3.0T scanner by assessing parameter reproducibility on free-breathing (FB) and respiratory-triggered (RT) sequences acquired with different numbers of signal averages (NSA).

Material and methods: In this prospective study 20 subjects (M/F: 10/10; age: 25-62 years, mean: 39 years) underwent IVIM magnetic resonance imaging (MRI) on a 3.0T scanner using an 18-channel phase-arrayed coil and four different echo-planar sequences, each with 10 b values: $0,10,30,50,75,100,150,200,500$, and $900 \mathrm{~s} / \mathrm{mm}^{2}$. Images were acquired with FB and RT with NSA = 1-4 (FBNSA1-4, RTNSA1-4) and with NSA = 3-6 (FBNSA3-6, RTNSA3-6). Subsequently, for the assessment of reproducibility of IVIM-derived parameters (f, D, D ), each subject was scanned again with an identical protocol during the same session. IVIM parameters were calculated. The distribution of IVIM-parameters for each DWI sequence were given as the median value with first and third quartile. Inter-scan reproducibility for each IVIM parameter was evaluated using coefficient of variance and Bland-Altman difference. Differences between FB sequence and RT sequence were tested using non-parametric Wilcoxon signed-rank test.

Results: Mean coefficient of variance (\%) for f, D, and $D^{*}$ ranged from 60 to 64, from 58 to 84, and from 82 to 99 for FBNSA1-4 sequence; from 50 to 69 , from 41 to 97 , and from 80 to 82 for RTNSA1-4 sequence; from 22 to 27,15 , and from 70 to 80 for FBNSA3-6 sequence; and from 21 to 32, from 12 to, and from 50 to 80 for RTNSA3-6 sequence, respectively.

Conclusions: Increasing the number of signal averages for IVIM acquisitions allows us to improve the reproducibility of IVIM-derived parameters. The sequence acquired during free-breathing with NSA = 3-6 was optimal in terms of reproducibility and acquisition time.

Key words: diffusion-weighted imaging, b-value, pseudo-diffusion, liver, intravoxel incoherent motion.

Correspondence address:

Joanna Podgórska, MD, PhD, Department of Radiology I, Maria Skłodowska-Curie Memorial Cancer Center and Institute of Oncology, 5 Roentgena St.,

02-781 Warsaw, Poland, phone: +48 22546 2280, fax: +48 225462307 , e-mail: jpodgo@gmail.com

Authors' contribution:

A Study design · B Data collection · C Statistical analysis · D Data interpretation · E Manuscript preparation · F Literature search · G Funds collection 


\section{Introduction}

There have been several attempts to use intravoxel incoherent motion (IVIM) diffusion-weighted imaging (DWI) for the characterisation of focal liver lesions and for the diagnosis of diffuse parenchymal liver disease [1-5]. However, due to significant differences in reported perfusion-related IVIM parameters in disease-free subjects, it is still not possible to establish normal values of perfusion fraction (f), which reflects the volume of flowing blood (range in published studies: from $19.0 \% \pm 5.5 \%$ to $32.16 \%$ $\pm 8.13 \%$ ) and the pseudo-diffusion coefficient $\left(\mathrm{D}^{\star}\right)$, which is presumed to show the velocity of capillary blood (range: from $39.61 \times 10^{-3} \mathrm{~mm}^{2} / \mathrm{s} \pm 12.34$ to $110.6 \times 10^{-3} \mathrm{~mm}^{2} / \mathrm{s} \pm 79$ ) [2-6]. Only the values of pure molecular diffusion (D) were similar in several publications, allowing more reliable comparison of this parameter between studies. Moreover, IVIM-derived parameters and maps are highly variable and inconsistent [7]. This inconsistency of IVIM technique could be related to different factors, including various distribution of applied b values, use of different diffusion gradient polarity (monopolar vs. bipolar gradients), or several motion correction methods, such as free-breathing (FB), breath-holding $(\mathrm{BH})$, and respiratory-triggering $(\mathrm{RT})$, and post-processing fitting methods $[4,8,9]$. Furthermore, acquired images usually are low quality, regardless of the type of applied sequence. The other important possible cause of diverse results as well as poor quality of IVIM images may be low signal-to-noise ratio (SNR). Poor SNR may lead to high, non-physiological heterogeneity among voxels, precluding reliable measurements. Even though several studies on the use of IVIM for assessment of hepatic disease have been published, only a few reports addressed the reproducibility of IVIM-derived parameters $[1,9,10]$. Lack of standardisation of IVIM technique and significant variance in calculated parameters among studies, thus far hamper the application of this method for routine MR liver imaging and for application of IVIM-derived parameters as eligible biomarkers.

Table 1. Numbers of signal averages (NSA) of diffusion-weighted imaging sequences

\begin{tabular}{|l|c|c|c|c|}
\hline $\mathbf{b}\left[\mathrm{s} / \mathrm{mm}^{2}\right]$ & FB NSA1-4 & RT NSA1-4 & FB NSA3-6 & RT NSA3-6 \\
\hline 0 & 1 & 1 & 3 & 3 \\
\hline 10 & 1 & 1 & 3 & 3 \\
\hline 30 & 1 & 1 & 3 & 3 \\
\hline 50 & 1 & 1 & 3 & 3 \\
\hline 75 & 1 & 1 & 3 & 3 \\
\hline 100 & 2 & 2 & 4 & 4 \\
\hline 150 & 2 & 2 & 4 & 4 \\
\hline 200 & 2 & 2 & 4 & 4 \\
\hline 500 & 2 & 2 & 4 & 4 \\
\hline 900 & 4 & 4 & 6 & 6 \\
\hline
\end{tabular}

FB - free-breathing, RT - respiratory-triggering
DWI of the liver on a $3.0 \mathrm{~T}$ scanner could be beneficial due to improved SNR compared to $1.5 \mathrm{~T}$ scanners; however, prominent magnetic susceptibility artefacts and distortion related to eddy currents remain a challenge and may lead to significant degradation of acquired images. Despite some publications regarding implementation of IVIM-derived parameters for the diagnosis of liver disease, there have been no reports addressing the reproducibility of this technique with different respiratory schemes at 3.0T MR systems.

To the best of our knowledge, there has been one study that addressed the effect of the number of signal averages (NSA) on the overall quality of IVIM images, SNR, and standard deviation (SD) of measurements [11], but none that focused on the effect on the reproducibility of the calculations. We hypothesised that the use of higher NSA could improve SNR, decrease SD of measurements, and possibly increase the reproducibility of calculated IVIM parameters. Therefore, the aim of this study was to optimise IVIM imaging of the liver on a 3.0T MR platform by assessing the reproducibility of IVIM parameters (f, D, $D^{*}$ ) for $\mathrm{FB}$ and RT sequences acquired with different numbers of signal averages.

\section{Material and methods}

The study was approved by the Institutional Review Board, and all volunteers participating in the study signed an informed consent form.

\section{Study population}

Between January 2017 and March 2017, 20 healthy volunteers were enrolled for this single-centre prospective study. Inclusion criteria were age $\geq 18$ years and willingness and ability to undergo MRI and participate in the study. Exclusion criteria were contraindications to MR imaging such as pacemakers, metal implants, and severe claustrophobia; age $<18$ years.

\section{Magnetic resonance imaging}

All MRI studies were performed at a 3T unit (Magnetom Skyra, Siemens Medical Solutions, Erlangen, Germany) using explorer gradients (maximum amplitude of $45 \mathrm{mT} / \mathrm{m}$ and slew rate of $200 \mathrm{mT} / \mathrm{m} / \mathrm{ms}$ ) and phased-array multicoil system (18 elements).

The examination protocol began with breath-hold (BH) T1-weighted gradient-echo (GRE) DIXON sequence and respiratory-triggered (RT) T2-weighted HASTE sequence, both performed in the axial plane. Then four echo-planar diffusion-weighted imaging (DWI) sequences in the axial plane were acquired with variable NSA. Two of them with single signal average for the five lowest $b$ values and four signal averages for the highest b: FB NSA1-4, RT NSA1-4, and two others with three signal averages 
Table 2. Acquisition parameters of applied diffusion-weighted imaging sequences

\begin{tabular}{|l|c|c|c|c|}
\hline Sequences & FB NSA1-4 & RT NSA1-4 & FB NSA3-6 & RT NSA3-6 \\
\hline Acquisition time & $1^{\prime} 58^{\prime \prime}$ & $3^{\prime} 48^{\prime \prime}-6^{\prime} 27^{\prime \prime}($ mean 5'04") & $4^{\prime}$ & $7^{\prime} 45^{\prime \prime}-16^{\prime} 05^{\prime \prime}\left(\mathrm{mean}^{\prime} 48^{\prime \prime}\right)$ \\
\hline Resolution matrix & $128 \times 84$ & $128 \times 84$ & $128 \times 84$ & $128 \times 84$ \\
\hline FoV read & 380 & 380 & 680 & 380 \\
\hline FoV phase & $65.6 \%$ & $65.6 \%$ & $5 \mathrm{~mm}$ & $5 \mathrm{~mm}$ \\
\hline Slice thickness & $5 \mathrm{~mm}$ & $5 \mathrm{~mm}$ & $35 \%$ & $35 \%$ \\
\hline Slice gap & $35 \%$ & $35 \%$ & 33 & 33 \\
\hline Slices & 33 & 33 & $6100 \mathrm{~ms}$ & $4200 \mathrm{~ms}$ \\
\hline TR & $6100 \mathrm{~ms}$ & $4200 \mathrm{~ms}$ & $56 \mathrm{~ms}$ & $56 \mathrm{~ms}$ \\
\hline TE & $56 \mathrm{~ms}$ & $56 \mathrm{~ms}$ & 3 & 3 \\
\hline Acceleration factor & 3 & 3 & & \\
\hline
\end{tabular}

NSA - numbers of signal averages, FB - free-breathing, RT - respiratory-triggering

Table 3. Mean regions of interest sizes for diffusion-weighted imaging sequences

\begin{tabular}{|l|c|c|c|c|c|c|c|c|}
\hline Sequences & \multicolumn{2}{|c|}{ FB NSA1-4 } & \multicolumn{2}{|c|}{ RT NSA1-4 } & \multicolumn{2}{c|}{ FB NSA3-6 } & \multicolumn{2}{c|}{ RT NSA3-6 } \\
\hline Examination & No.1 & No.2 & No.1 & No.2 & No.1 & No.2 & No.1 & No.2 \\
\hline Mean value $\left(\mathrm{cm}^{2}\right)$ & 1.35 & 1.59 & 1.28 & 1.31 & 1.32 & 1.29 & 1.39 & 1.58 \\
\hline Standard deviation $\left(\mathrm{cm}^{2}\right)$ & 0.62 & 0.52 & 0.56 & 0.53 & 0.54 & 0.47 & 0.56 & 0.58 \\
\hline
\end{tabular}

NSA - numbers of signal averages, FB - free-breathing, RT - respiratory-triggering

for the five lowest $b$ values and six signal averages for the highest b: FB NSA3-6, RT NSA3-6 (Table 1). Selected parameters of applied DWI sequences are shown in Table 2. All DWI sequences were acquired with the same $10 \mathrm{~b}$ values: $0,10,30,50,75,100,150,200,500,900 \mathrm{~s} / \mathrm{mm}^{2}$ but different NSA (NSA for each b value for all DWI sequences are presented in Table 1).

After conclusion of the whole MR imaging protocol, each patient was moved out of the MRI unit and instantly positioned again inside the magnet. Then four DWI sequences were acquired for a second time with the same parameters, to evaluate the reproducibility of IVIM parameters.

The acquisition/mean acquisition time for different DWI sequences ranged from $1 \mathrm{~min} 58 \mathrm{sec}$ (for FB NSA1-4) to 9 min $48 \mathrm{sec}$ (for RT NSA3-6).

\section{Image analysis}

All measurements were performed at commercial workstations (Syngovia, Siemens Medical Solution, Erlangen) by two radiologists (JP and AAG) with six and four years of experience in body MR imaging, respectively. For the calculation of IVIM parameters $\left(\mathrm{f}, \mathrm{D}\right.$, and $\mathrm{D}^{*}$ ) regions of interest (ROIs) were drawn on chosen slices of $b=0$ images, independently for each DWI sequence for the right liver lobe. Then ROIs were copied and pasted from these images to corresponding scans acquired with higher b values. Areas containing blood vessels and artefacts were avoided. Measurements of signal intensity (SI) were performed twice and averaged. We did not include the left liver lobe in the analysis due to the presence of artefacts related to cardiac motion. Table 3 shows the mean ROI sizes for different DWI sequences, separately for the first (No. 1) and second acquisition (No. 2).

Estimated signal-to-noise ratio (eSNR) was calculated for four b values $\left(0,30,200,900 \mathrm{~s} / \mathrm{mm}^{2}\right)$ according to the following formula:

$$
\mathrm{eSNR}=\frac{\mathrm{SI}_{\text {means }}}{\mathrm{SD}_{\text {noise }}}
$$

where $\mathrm{SI}_{\text {means }}$ is mean signal intensity, and $\mathrm{SD}_{\text {noise }}$ is standard deviation of background noise (measured on ROI placed in the background).

IVIM parameters were calculated using the IVIM model equations described by Le Bihan et al. [12].

$$
\frac{\mathrm{S}}{\mathrm{S}_{0}}=(1-\mathrm{f}) \cdot \exp (-\mathrm{b} \cdot \mathrm{D})+\mathrm{f} \cdot \exp \left(-\mathrm{b} \cdot\left(\mathrm{D}^{\star}+\mathrm{D}\right)\right)
$$

$S$ - signal intensity in the pixel with diffusion gradient $b$, $\mathrm{S}_{0}$ - signal intensity in the pixel without diffusion gradient,

$\mathrm{D}$ - true diffusion as reflected by pure molecular diffusion, $\mathrm{f}$ - fractional perfusion related to microcirculation,

$\mathrm{D}^{*}$ - pseudo-diffusion coefficient representing perfusion-related diffusion or incoherent microcirculation.

For calculations we applied our own program written in Gnuplot program version 5.0, patchlevel 4, utilising the method of the nonlinear least-squares (NLLS) Marquardt-Levenberg algorithm. 
Table 4. Intravoxel incoherent motion-derived parameter values of the liver

\begin{tabular}{|c|c|c|c|c|c|c|}
\hline \multirow[t]{2}{*}{ Parameter } & \multicolumn{2}{|c|}{ First examination } & \multicolumn{2}{|c|}{ Second examination } & \multicolumn{2}{|c|}{$p$} \\
\hline & $\begin{array}{l}\text { FB, median } \\
\text { (quartiles) }\end{array}$ & $\begin{array}{l}\text { RT, median } \\
\text { (quartiles) }\end{array}$ & $\begin{array}{l}\text { FB, median } \\
\text { (quartiles) }\end{array}$ & $\begin{array}{l}\text { RT, median } \\
\text { (quartiles) }\end{array}$ & $\mathrm{FB}$ & RT \\
\hline \multicolumn{7}{|c|}{ NSA1-4 } \\
\hline$f(\%)$ & $28(25-34)$ & $28(25-31)$ & $26(0.22-0.30)$ & $25(0.19-0.30)$ & 0.50 & 0.28 \\
\hline $\mathrm{D}\left(\times 10^{-3} \mathrm{~mm}^{2} / \mathrm{s}\right)$ & $0.90(0.76-1.06)$ & $1.00(0.89-1.16)$ & $0.99(0.90-1.21)$ & $1.02(0.92-1.15)$ & 0.05 & 0.42 \\
\hline$D^{*}\left(\times 10^{-3} \mathrm{~mm}^{2} / \mathrm{s}\right)$ & $44(8-120)$ & $78(29-115)$ & $128(23-185)$ & $76(38-152)$ & 0.06 & 0.23 \\
\hline \multicolumn{7}{|c|}{ NSA3-6 } \\
\hline$f(\%)$ & $24(18-28)$ & $21(16-24)$ & $22(19-26)$ & $24(20-26)$ & 0.66 & 0.16 \\
\hline $\mathrm{D}\left(\times 10^{-3} \mathrm{~mm}^{2} / \mathrm{s}\right)$ & $1.01(0.97-1.13)$ & $1.03(0.99-1.14)$ & $1.07(0.99-1.14)$ & $1.06(0.98-1.28)$ & 0.39 & 0.45 \\
\hline$D^{*}\left(\times 10^{-3} \mathrm{~mm}^{2} / \mathrm{s}\right)$ & 78 (49-117) & 89 (49-114) & $66(42-123)$ & 67 (44-111) & 0.97 & 0.62 \\
\hline
\end{tabular}

NSA - numbers of signal averages, $\mathrm{FB}$ - free-breathing, RT - respiratory-triggering

\section{Statistical analysis}

All statistical analysis was performed using $\mathrm{R}$ version 3.3.2. The distribution of extracted IVIM-parameters for each DWI sequence was given as the median value with first and third quartile. The calculated values $\mathrm{f}, \mathrm{D}$, and $\mathrm{D}^{*}$ were compared between specific group pairs (FB in the first examination vs. FB in the second examination; RT in the first examination vs. RT in the second examination). A non-parametric Wilcoxon signed-rank test was used. Statistical significance was defined as $p<0.05$. Normal distribution of difference between FB1 vs. FB2 and RT1 vs. RT2 was tested using Shapiro-Wilk test. Inter-examination repeatability between FB and $\mathrm{RT}$ of $\mathrm{f}, \mathrm{D}$, and $\mathrm{D}^{*}$ were assessed by coefficient of variation $(\mathrm{CV})$ and Bland-Altman mean difference and 95\% limits of agreements (BA-LA).

\section{Results}

\section{Intravoxel incoherent motion parameters}

Table 4 shows values of calculated IVIM parameters of the right liver lobe for applied DWI sequences. Calculated values of $f, D$, and $D^{*}$ were not significantly different between FB and RT sequences for the first and second examinations.

Figure 1 shows distribution of IVIM parameters in healthy volunteers, obtained with the use of all applied DWI sequences for the first (No. 1) and second (No. 2) acquisition. The horizontal line in each box represents the median. Top and bottom of boxes represent $25 \%$ and $75 \%$ percentiles of data values, respectively. Individual points plotted on the figure represent outliers.

\section{Median values of IVIM-derived parameters of the liver}

The mean IVIM-derived parameters were calculated as a median value of two consecutive acquisitions for each type of sequence. Using the most reproducible DWI se- quence (FB NSA3-6), the following numbers were obtained: $\mathrm{f}=23 \%, \mathrm{D}=1.03 \times 10^{-3} \mathrm{~mm}^{2} / \mathrm{s}, \mathrm{D}^{*}=72 \times 10^{-3} \mathrm{~mm}^{2} / \mathrm{s}$. Similar values were obtained with RT NSA3-6 sequence: $\mathrm{f}=22 \%, \mathrm{D}=1.07 \times 10^{-3} \mathrm{~mm}^{2} / \mathrm{s}, 83 \times 10^{-3} \mathrm{~mm}^{2} / \mathrm{s}$. Corresponding values differed for FB NSA1-4 acquisition ( $\mathrm{f}=27 \%, \mathrm{D}=0.98 \times 10^{-3} \mathrm{~mm}^{2} / \mathrm{s}, \mathrm{D}^{*}=56 \times 10^{-3} \mathrm{~mm}^{2} / \mathrm{s}$ ) and for RT NSA1-4 acquisition ( $\mathrm{f}=27 \%, \mathrm{D}=1.01 \times 10^{-3}$ $\left.\mathrm{mm}^{2} / \mathrm{s}, \mathrm{D}^{\star}=78 \times 10^{-3} \mathrm{~mm}^{2} / \mathrm{s}\right)$.

\section{Reproducibility}

Coefficient of variance (CoV) for FB NSA1-4, RT NSA1-4, FB NSA3-6, and RT NSA3-6 sequences varied from 12\% to $99 \%$ (Table 5). The coefficient of variance of IVIM-derived parameters of the liver was better for NSA3-6 sequences, compared to NSA1-4 sequences (except for D* calculated from RT NSA3-6 acquisition). The comparison between free-breathing and respiratory-triggered acquisitions demonstrated that respiratory-triggering generated better inter-scan reproducibility for NSA1-4 sequences, whereas free-breathing acquisition was more repeatable in terms of IVIM-derived parameters for NSA3-6 sequences.

Bland-Altman plots of inter-examination repeatability of IVIM parameters for NSA 3-6 sequence are shown in Figure 2. Bland-Altman plots for NSA1-4 sequences were not presented due to the fact that data did not fulfil the requirement of the normal distribution. For NSA3-6 FB sequence all the data points of $f, D$, and $D^{*}$ were within the limits of agreements. For NSA3-6 RT sequence only $\mathrm{D}$ and $\mathrm{D}^{\star}$ were within the limits of agreement. The limits of agreement for $\mathrm{f}, \mathrm{D}$, and $\mathrm{D}^{\star}$ parameters were lower for FB sequence (Table 6).

\section{Signal-to-noise ratio}

Signal-to-noise ratios of the liver for selected b value images $(0,30,200,900)$ for all applied DWI sequences are shown in Table 7. In all acquisitions and for all analysed b values the average eSNR was above 30 . In both types 

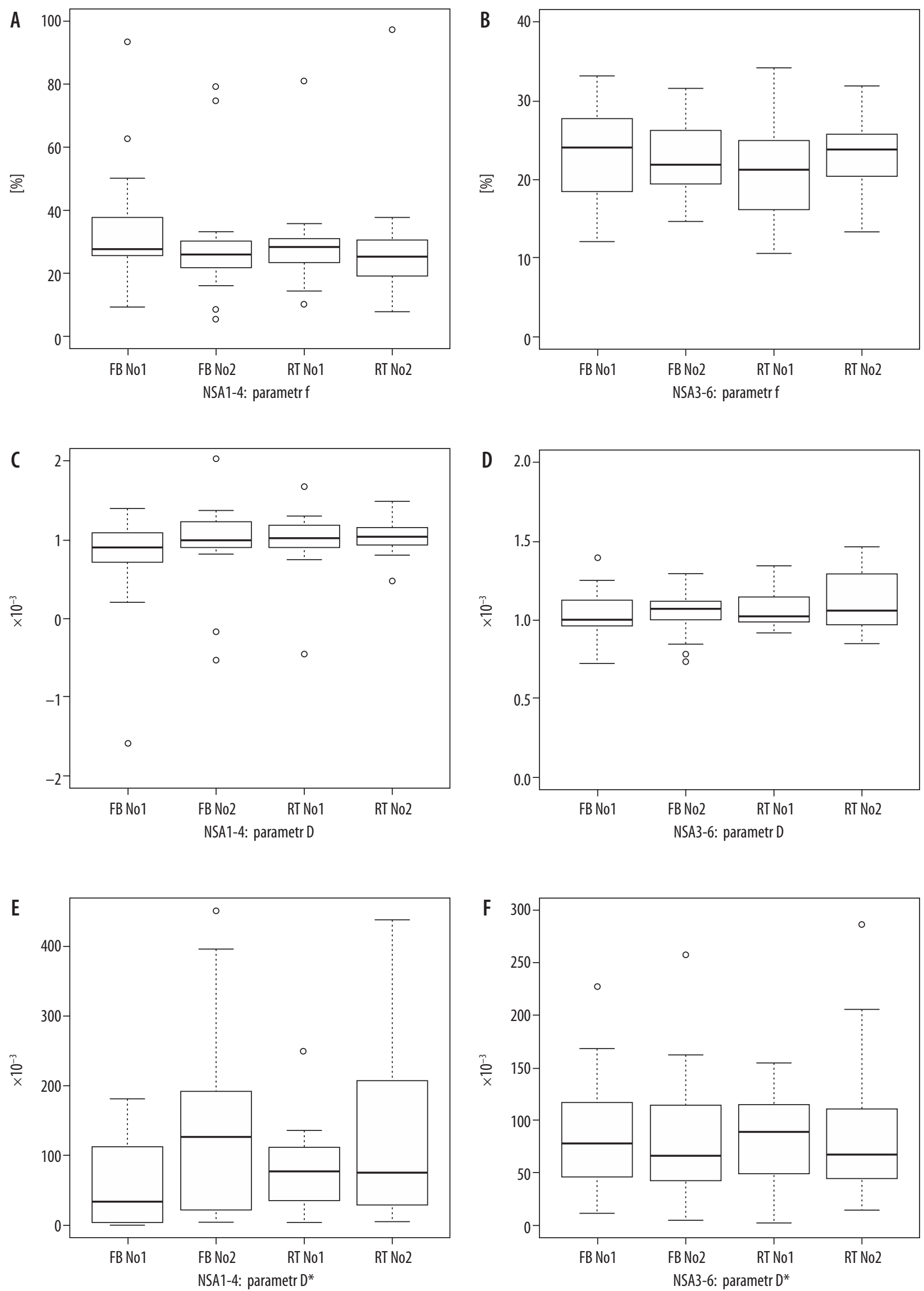

Figure 1. Box-plots of $f, D$, and $D^{*}$ values 
Table 5. Coefficient of variation (expressed in \%) of intravoxel incoherent motion parameters

\begin{tabular}{|c|c|c|c|c|}
\hline & \multicolumn{2}{|c|}{ First examination } & \multicolumn{2}{|c|}{ Second examination } \\
\hline & FB & RT & FB & RT \\
\hline & \multicolumn{4}{|c|}{ NSA1-4 } \\
\hline $\mathrm{f}[\%]$ & 60 & 50 & 64 & 69 \\
\hline $\mathrm{D}\left[\times 10^{-3} \mathrm{~mm}^{2} / \mathrm{s}\right]$ & 84 & 41 & 58 & 97 \\
\hline \multirow[t]{2}{*}{$D^{*}\left[\times 10^{-3} \mathrm{~mm}^{2} / \mathrm{s}\right]$} & 99 & 82 & 99 & 88 \\
\hline & \multicolumn{4}{|c|}{ NSA3-6 } \\
\hline $\mathrm{f}[\%]$ & 27 & 32 & 22 & 21 \\
\hline $\mathrm{D}\left[\times 10^{-3} \mathrm{~mm}^{2} / \mathrm{s}\right]$ & 15 & 12 & 15 & 17 \\
\hline$D^{*}\left[\times 10^{-3} \mathrm{~mm}^{2} / \mathrm{s}\right]$ & 70 & 50 & 80 & 80 \\
\hline
\end{tabular}

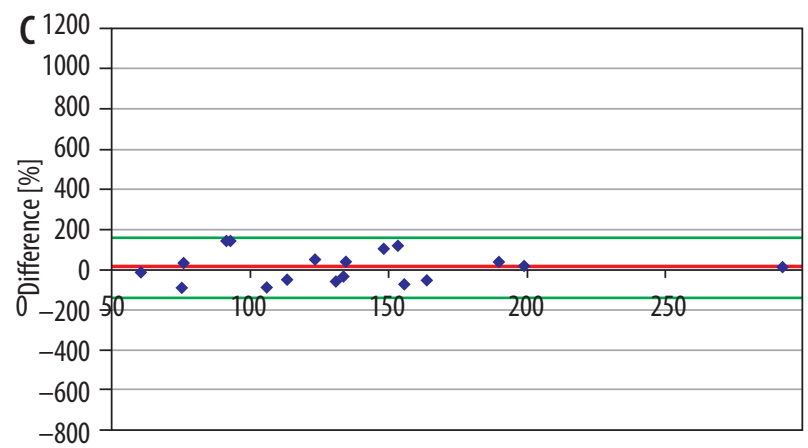

Average

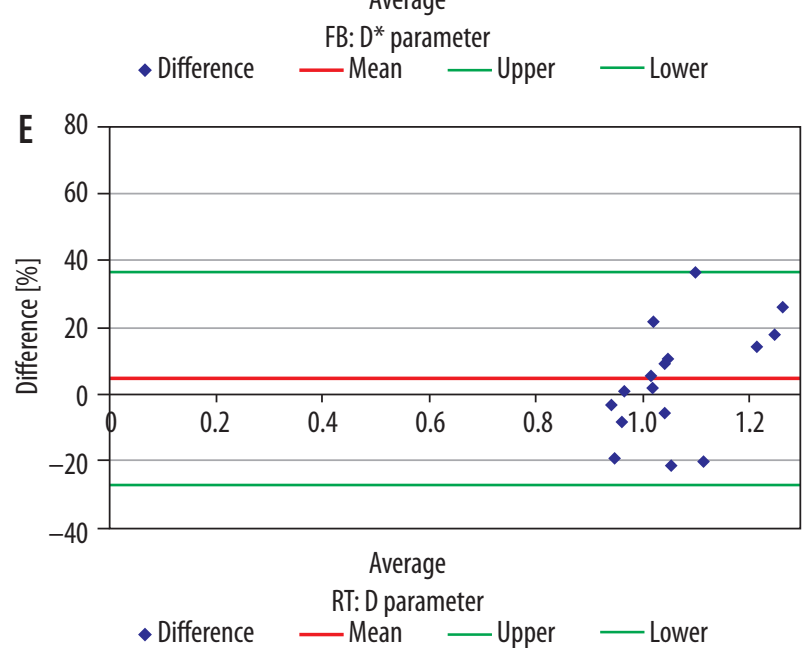

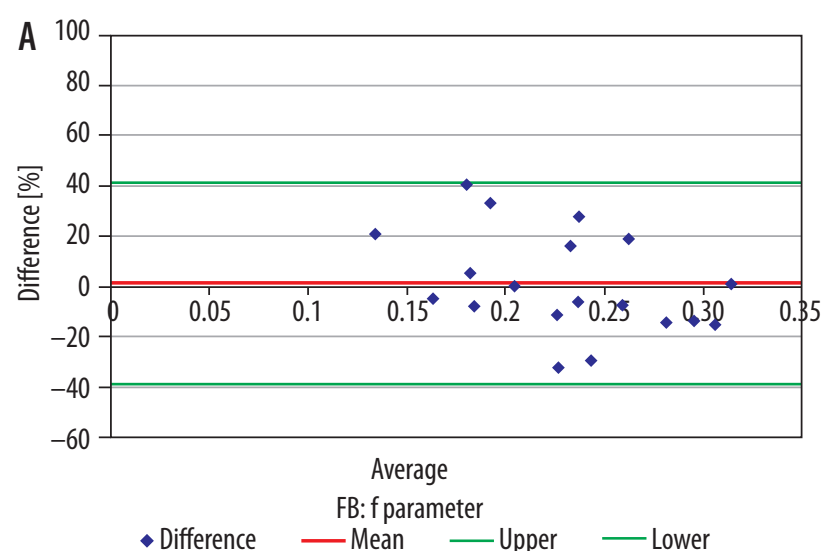

of acquisitions (NSA1-4 vs. NSA3-6) eSNRs were higher (for all b values) in respiratory-triggered sequences. The eSNRs were higher for NSA3-6 sequences compared to NSA1-4 sequences, except for RT acquisition with a b value of 200 (mean eSNR was higher for NSA1-4 sequence) and FB acquisition with a b value of 900 (mean eSNR was equal for both acquisitions).

\section{Discussion}

IVIM DW model, introduced in the late 1980s by Le Bihan et al. [12], is an imaging technique employing multiple b values and bi-exponential fitting for the simultaneous estimation of the pure molecular water diffusion and micro-
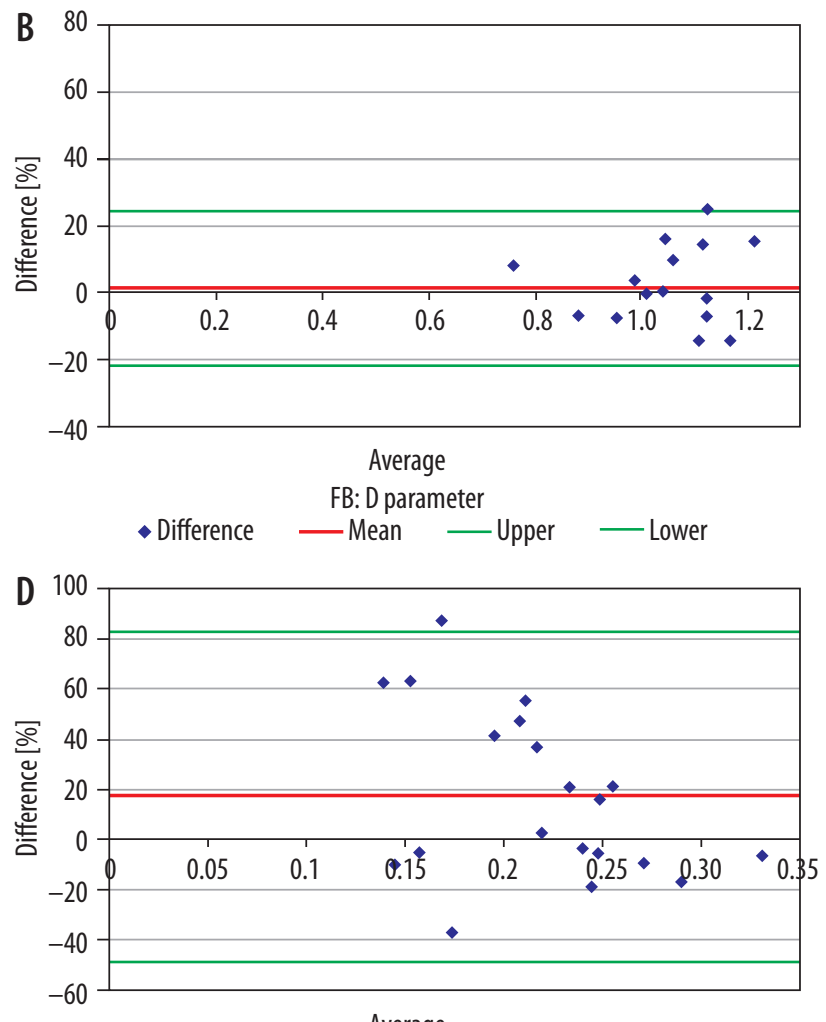

Average

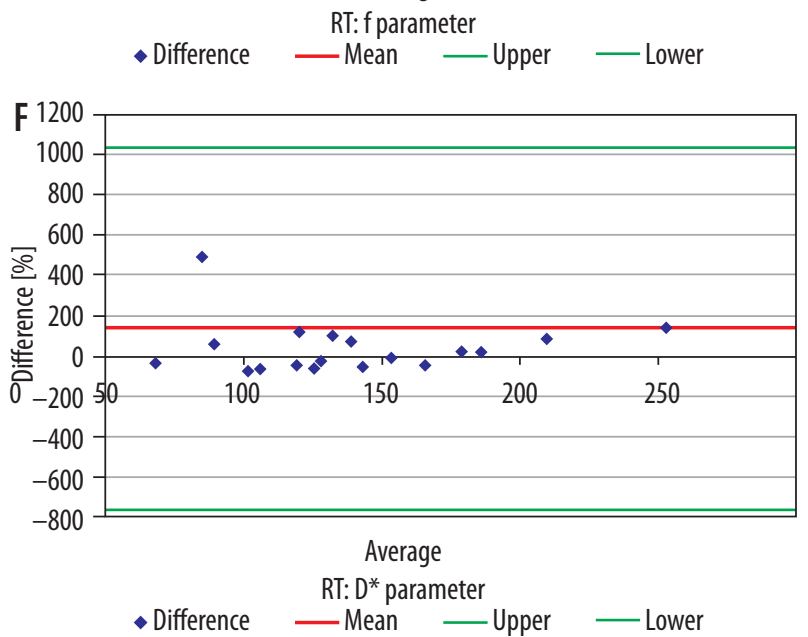

Figure 2. Bland-Altman plot of percentage difference against mean measurements, with mean percentage difference and upper and lower limits of agreements 
circulation of blood in the capillaries (perfusion). Interest in the application of IVIM technique for the imaging of abdominal organs has recently increased, including diagnosis of liver, pancreatic, and renal diseases. The possible advantage of this technique is quantitative assessment of both tumour (or tissue) cellularity and vascularity, without the need for intravenous contrast material administration. However, there are several important limitations preventing the application of IVIM analysis in routine practice. Among the most important ones are the lack of standardisation of IVIM sequences, prolonged acquisition time (due to application of multiple b values), relatively poor quality of obtained images, and predilection of some of the IVIM-derived parameters (particularly $\mathrm{D}^{\star}$ ) to errors.

Because IVIM imaging relies on quantitative analysis of $f, D$, and $D^{*}$, reproducibility of calculated IVIM parameters remains a crucial issue. For this reason, it is necessary to standardise both the imaging protocol and analysis of acquired data. Previous optimisation studies mostly concentrated on several issues including choice of $b$ values, different respiratory correction schemes, and postprocessing methods. To the best of our knowledge, our study is the first attempt to systematically evaluate the effect of the number of signal averages on the reproducibility of IVIM-derived parameters. For this purpose, we compared consecutive acquisitions obtained during the same session, with the shortest possible interval between them. In previous studies evaluating the reproducibility of IVIM acquisitions the average delays between two MR examinations were nine days (range: 0-45 days) and 13 days (range: $5-45$ days) $[6,10]$. We believe that the method utilised in our study enabled us to diminish possible substantial changes related to altered physiological perfusion in the human body.

In published reports, NSA for low b values varied from 1 to 3 and in some reports this figure was not available $[6,10,13,14]$. The new generation of MRI scanners permits separate adjustment of NSA for each b value, thus enabling further compensation of signal loss on high $b$ value images by increasing the NSA. We utilised this approach by employing increasing NSA: from 1 to 4 for $\mathrm{FB}$ and from 3 to 6 for RT sequences (Table 2). Our results showed that utilisation of single signal average (for low b values) for IVIM imaging is not sufficient to obtain reliable results. Increasing NSA allows us to improve the global inter-scan reproducibility of IVIM-derived parameters for both free-breathing and respiratory-triggered sequences. This improvement was most noticeable for FB technique for parameter $\mathrm{D}^{*}$ - decrease of mean coefficient of variance from $99 \%$ for FB NSA1-4 to $70 \%$ (in the first examination) and $80 \%$ (in the second examination) for FB NSA3-6, which is particularly prone to miscalculation.

Interestingly, in terms of inter-scan reproducibility the FB NSA3-6 sequence performed better than RT NSA3-6 sequence. The limits of agreements between the paired measurements for $\mathrm{f}, \mathrm{D}$, and $\mathrm{D}^{\star}$ parameters were lower
Table $6.95 \%$ confidence intervals of percentage difference between the paired measurements for NSA 3-6

\begin{tabular}{|l|c|c|}
\cline { 2 - 3 } & \multicolumn{2}{c}{ NSA3-6 } \\
\cline { 2 - 3 } & $\mathrm{FB}(\%)$ & RT $(\%)$ \\
\hline $\mathrm{D}[\%]$ & $-39 ; 41$ & $-49 ; 83$ \\
\hline$D^{*}\left[\times 10^{-3} \mathrm{~mm}^{2} / \mathrm{s}\right]$ & $-22 ; 25$ & $-27 ; 36$ \\
\hline
\end{tabular}

Wider intervals indicate poorer measurement reproducibility.

NSA - numbers of signal averages, FB - free-breathing, RT - respiratory-triggering

Table 7. Estimated signal-to-noise ratio of the liver for selected b value images (shown as mean value \pm standard deviation)

\begin{tabular}{|l|c|c|c|c|}
\hline $\boldsymbol{b}$-value & FB NSA1-4 & RT NSA1-4 & FB NSA3-6 & RT NSA3-6 \\
\hline 0 & $65 \pm 44$ & $94 \pm 76$ & $78 \pm 59$ & $99 \pm 77$ \\
\hline 30 & $55 \pm 44$ & $63 \pm 48$ & $62 \pm 40$ & $74 \pm 53$ \\
\hline 200 & $43 \pm 34$ & $66 \pm 59$ & $51 \pm 37$ & $61 \pm 43$ \\
\hline 900 & $35 \pm 41$ & $37 \pm 35$ & $35 \pm 36$ & $40 \pm 34$ \\
\hline
\end{tabular}

NSA - numbers of signal averages, FB - free-breathing, RT - respiratory-triggering

for FB sequence (Table 6). This difference was again most prominent for $\mathrm{D}^{\star}$. This finding is in opposition to a previous publication of Dyvorne et al., who concluded that RT sequence yielded the best image quality, reproducibility, and ability to discriminate between fibrotic and healthy liver, whereas stronger image blurring was noted on FB acquisitions [10]. They reported no significant difference in $\mathrm{D}^{\star}$ between healthy volunteers and HCV patients, attributing it to high uncertainty and poor reproducibility of $\mathrm{D}^{\star}$. In our group of volunteers, the reproducibility of $\mathrm{D}^{*}$ was better using FB NSA3-6 acquisition than using RT NSA3-6 acquisition. We did not perform visual, qualitative evaluation of the of IVIM images because we believe that quantitative assessment of IVIM-derived parameters is the most important. The possible reason for better performance of FB NSA3-6 over RT NSA3-6 acquisition may be the irregular pattern of respiration encountered in some volunteers. It could cause, along with possibly imperfect system of navigator echo gating, suboptimal acquisition of respiratory-triggered images. Moreover, the mean acquisition time of RT NSA3-6 sequence (9'48"; range: $\left.7^{\prime} 45^{\prime \prime}-16^{\prime} 05^{\prime \prime}\right)$ was over double that of the equivalent free-breathing sequence (4'). We cannot exclude that it may further increase patients' discomfort during examination and diminish their cooperation. In addition, prolonged acquisition time of IVIM sequence leads to extension of overall imaging time and disturbs the workflow in daily clinical practice.

In published studies regarding IVIM MR imaging of the liver, different respiratory schemes were employed, including free-breathing $[5,6,10,13,14]$, breath-holding [9], and respiratory-triggering $[6,10,15]$. These studies were performed on both $1.5 \mathrm{~T}[1,6,7]$ and $3.0 \mathrm{~T}$ systems $[3,6,15]$. 
Dyvorne et al., who compared RT and FB IVIM DWI sequences on a $1.5 \mathrm{~T}$ system, concluded that RT sequence with bipolar diffusion-encoding schemes demonstrated the highest image quality and reproducibility [10]. These results are contrary to our findings, although this difference may be attributed to different field strengths of MR scanners used in these studies. To the best of our knowledge, our publication is the first addressing reproducibility of IVIM DWI sequences with different respiratory schemes for liver imaging on a 3.0T MR system.

3.0T DWI of the liver appears to be an interesting option, due to improved SNR compared to $1.5 \mathrm{~T}$ scanners. However, it remains challenging because of prominent magnetic susceptibility artefacts and distortion related to eddy currents [16], and most researchers utilise $1.5 \mathrm{~T}$ platforms. IVIM imaging of the liver is even more demanding because it is a moving organ, prone to motion-induced miscalculations. Regarding ADC values, Rosenkrantz et al. [17] reported similar results for both $1.5 \mathrm{~T}$ and 3.0T systems, whereas Dale et al. [18] found significant differences in ADC values for these two field strengths. Theoretically, IVIM-derived parameters may provide more accurate data on true molecular movement of water (diffusion), along with microcirculation of blood in the capillaries (perfusion). However, the knowledge of the average values of IVIM-derived parameters of normal liver for both 1.5T and 3.0T units is critical for the diagnosis and assessment of severity of hepatic disease. Otherwise, it cannot be used as an eligible, quantitative biomarker for the estimation of response to therapy in prospective drug trials.

In our study of 20 healthy volunteers the median $D$ value $\left(1.03 \pm 0.18 \times 10^{-3} \mathrm{~mm}^{2} / \mathrm{s}\right)$ calculated from optimal sequence (FB NSA3-6) was similar to values obtained by other researchers $[6,13,15]$ utilising $3.0 \mathrm{~T}$ systems. The difference in median f obtained in our study $(23 \% \pm 7 \%)$ and in previous publications $(26.9 \% \pm 8.8 \% ; 19.0 \% \pm 5.5 \%$ - on $3.0 \mathrm{~T}$ and $30.8 \% \pm 4.95 \%$ - on $1.5 \mathrm{~T}$ ) was moderate, whereas the discrepancy in average $\mathrm{D}^{*}$ between different studies was more prominent $\left(72 \pm 61 \times 10^{-3} \mathrm{~mm}^{2} / \mathrm{s}\right.$ in our study and $110.6 \pm 79,57.2 \pm 40.2-$ on $3.0 \mathrm{~T}$ and $59.67 \pm$ 12.34 - on $1.5 \mathrm{~T}$, in previous reports) $[6,13,15]$. This data, along with considerable inter-scan reproducibility for $\mathrm{D}^{*}$, confirms that the pseudo-diffusion coefficient is burdened with a larger error. Consequently, precise assessment of this parameter requires further research in terms of the standardisation and optimisation of image acquisition as well as postprocessing. Moreover, a lack of standardisation of IVIM technique and significant variance in calculated parameters among studies thus far precludes application of this method for routine MR liver imaging for application as an eligible biomarker. In addition, IVIM-derived parameters, similarly to ADC values, are possibly both vendor and field strength dependent (1.5T vs. 3.0T) $[19,20]$. Among many factors that may influence DWI calculations are echo time, gradient amplitude, and separation of diffusion gradient [20]. Accurate calculation of IVIM parameters from a 3.0T MR scanner requires high SNR for all acquired $b$ values. Our study suggests that application of minimum NSA $=3$ for low $b$ values (with progressive increase up to 6 for the highest b value) is required to get reproducible IVIM calculations.

DWI sequences are nowadays incorporated into routine MRI protocols of the liver and utilised for the diagnosis of both focal and diffuse hepatic disease. Quantitative assessment of apparent diffusion coefficient (ADC) facilitated discrimination of malignant from benign focal liver lesions (FLLs) and by some researchers is advocated for evaluation of diffuse liver disease. Supposedly, IVIM-derived parameters, such as perfusion fraction (f), pseudodiffusion coefficient $\left(\mathrm{D}^{*}\right)$, and true diffusion coefficient (D) enable greater insight into the physiology of liver tissue compared to ADC alone. This hypothesis was confirmed by several reports $[1-4,10,13,15]$. Yoon et al., who evaluated FLLs on a 3.0T system using IVIM-derived parameters, concluded that D provided better diagnostic performance than ADC in discrimination of benign and malignant FLLs, whereas $\mathrm{D}^{\star}$ and $\mathrm{f}$ were significantly higher in hypervascular lesions [13]. The decrease of $\mathrm{D}^{*}$ was reported most frequently in liver steatosis [5], fibrosis, and cirrhosis $[2,4,13]$. The decline of $\mathrm{D}[2,5]$ and $\mathrm{f}[2,10]$ was also reported as a valuable indicator of diffuse liver disease in human studies.

Our study has some limitations. First, the number of studied subjects was limited to 20 and the mean age of this group was relatively low. Second, we applied only $10 \mathrm{~b}$ values for IVIM acquisitions. The utilisation of more $b$ values could result in more accurate calculation of IVIM-derived parameters but at the expense of considerable prolongation of imaging time. Our imaging protocol was a trade-off between accuracy and reasonable scanning time. Similarly to some other researchers, we assumed that the number of less than initially suggested $16 \mathrm{~b}$ values enables acquisition of reliable data $[4,13]$. Application of more $b$ values would prolong examination time and could be difficult to accept in clinical practice. Third, we did not include the left liver lobe in our measurements and analysis due to significant cardiac motion artefacts in this area.

In conclusion, increasing the number of signal averages for IVIM acquisitions allows a decrease the reproducibility error of IVIM-derived parameters on 3.0T systems. The sequence acquired during free-breathing with NSA rising from 3 (for low $b$ values) to 6 (for the highest $b$ value) was optimal in terms of both reproducibility and acquisition time.

\section{Conflict of interest}

The authors report no conflict of interest. 


\section{References}

1. Dyvorne H, Jajamovich G, Kakite S, et al. Intravoxel incoherent motion diffusion imaging of the liver: Optimal b-value subsampling and impact on parameter precision and reproducibility. Eur J Radiol 2014; 83: 2109-2113.

2. Patel J, Sigmund EE, Rusinek H, et al. Diagnosis of cirrhosis with intravoxel incoherent motion diffusion MRI and dynamic contrast-enhanced MRI alone and in combination: preliminary experience. J Magn Reson Imaging 2010; 31: 589-600.

3. Yoon JH, Lee JM, Baek JH, et al. Evaluation of hepatic fibrosis using intravoxel incoherent motion in diffusion-weighted liver MRI. J Comput Assist Tomogr 2014; 38: 110-116.

4. Luciani A, Vignaud A, Cavet M, et al. Liver cirrhosis: intravoxel incoherent motion MR imaging - pilot study. Radiology 2008; 249: 891-899.

5. Guiu B, Petit J-M, Capitan V, et al. Intravoxel incoherent motion diffusion-weighted imaging in nonalcoholic fatty liver disease: A 3.0-T MR study. Radiology 2012; 265: 96-103.

6. Cui Y, Dyvorne H, Besa C, et al. IVIM diffusion-weighted imaging of the liver at 3.0 T: Comparison with 1.5 T. Eur J Radiol Open 2015; 2 : 123-128.

7. Lemke A, Stieltjes B, Schad LR, et al. Toward an optimal distribution of $b$ values for intravoxel incoherent motion imaging. Magn Reson Imaging 2011; 29: 766-776.

8. Yamada I, Aung W, Himeno Y, et al. Diffusion coefficients in abdominal organs and hepatic lesions: evaluation with intravoxel incoherent motion echo-planar MR imaging. Radiology 1999; 210: 617-623.

9. Lemke A, Laun FB, Klauß M, et al. Differentiation of pancreas carcinoma from healthy pancreatic tissue using multiple b-values: Comparison of apparent diffusion coefficient and intravoxel incoherent motion derived parameters. Invest Radiol 2009; 44: 769-775.

10. Dyvorne HA, Galea N, Nevers T, et al. Diffusion-weighted imaging of the liver with multiple b values: effect of diffusion gradient polarity and breathing acquisition on image quality and intravoxel incoherent motion parameters - a pilot study. Radiology 2013; 266: 920-929.
11. Leporq B, Saint-Jalmes H, Rabrait C, et al. Optimization of intravoxel incoherent motion imaging at 3.0 Tesla for fast liver examination. J Magn Reson Imaging 2015; 41: 1209-1217.

12. Le Bihan D, BretonE, Lallemand D, et al. Separation of diffusion and perfusion in intravoxel incoherent motion MR imaging. Radiology 1988; 168: 497-505.

13. Yoon JH, Lee JM, Yu MH, et al. Evaluation of hepatic focal lesions using diffusion-weighted MR imaging: Comparison of apparent diffusion coefficient and intravoxel incoherent motion-derived parameters. J Magn Reson Imaging 2014; 39: 276-285.

14. Yoon JH, Lee JM, Baek JH, et al. Evaluation of hepatic fibrosis using intravoxel incoherent motion in diffusion-weighted liver MRI. J Magn Reson Imaging 2014; 38: 110-116.

15. Guiu B, Petit J-M, Capitan V, et al. Intravoxel incoherent motion diffusion-weighted imaging in nonalcoholic fatty liver disease: A 3.0-T MR study. Radiology 2012; 265: 96-103.

16. Springer F, Martirosian P, Boss A, et al. Current problems and future opportunities of abdominal magnetic resonance imaging at higher field strengths. Top Magn Reson Imaging 2010; 21: 141-148.

17. Rosenkrantz AB, Oei M, Babb JS, et al. Diffusion-weighted imaging of the abdomen at 3.0 Tesla: Image quality and apparent diffusion coefficient reproducibility compared with 1.5 Tesla. J Magn Reson Imaging 2011; 33: 128-135.

18. Dale BM, Braithwaite AC, Boll DT, et al. Field strength and diffusion encoding technique affect the apparent diffusion coefficient measurements in diffusion-weighted imaging of the abdomen. Invest Radiol 2010; 45: 104-108.

19. Barbieri S, Donati OF, Froehlich JM, et al. Comparison of intravoxel incoherent motion parameters across MR imagers and field strengths: evaluation in upper abdominal organs. Radiology 2016; 279: 784-794.

20. Zhu J, Zhang J, Gao J, et al. Apparent diffusion coefficient normalization of normal liver: Will it improve the reproducibility of diffusion-weighted imaging at different MR scanners as a new biomarker? Medicine (Baltimore) 2017; 96: e5910. 